Dialectologia. Special issue, IX (2021), 135-149.

ISSN: 2013-2247

Received 21 April 2021.

Accepted 30 April 2021.

\title{
EL ARCO IRIS EN EL ATLAS LINGÜÍSTICO DE LA PENÍNSULA IBÉRICA ${ }^{1}$
}

\author{
Pilar GARCíA MOUTON \\ ILLA, CSIC * \\ pilar.garciamouton@cchs.csic.es
}

Resumen

Este trabajo tiene como objetivo valorar la aportación del $A L P I$ al estudio de las denominaciones peninsulares del arco iris y su dimensión etnográfica a través de la comparación de los atlas lingüísticos en sus diferentes cronologías.

\section{Palabras clave}

arco iris, $A L P I$, refranes meteorológicos, geolingüística, etnografía

\section{RAINBOW IN THE ATLAS LINGÜÍSTICO DE LA PENÍNSULA IBÉRICA}

\section{Abstract}

This work aims to assess the contribution of the $A L P I$ to the study of peninsular denominations of the rainbow and their ethnographic dimension through the comparison of linguistic atlases in their different chronologies.

\section{Keywords}

rainbow, $A L P I$, meteorological proverbs, geolinguistics, ethnography

\footnotetext{
${ }^{1}$ En la página $<A L P I . c s i c . e s / e s / c o n s u l t a>$ se puede acceder a los datos de 103 preguntas del cuestionario del $A L P I$, elaboradas y editadas en el marco de los proyectos que desde hace años desarrollamos Pilar García Mouton (coord.), Inés Fernández-Ordóñez, Maria-Pilar Perea, João Saramago y Xulio Sousa, integrados en el proyecto coordinado PGC2018-095077-B-C41/C42/C43/C44, El Atlas Lingüístico de la Península Ibérica: edición digital y análisis de datos.

*Albasanz, 26-28, 1E15. 28037 Madrid (España).
} 


\section{Introducción}

A partir de los atlas regionales españoles, ${ }^{2}$ y de atlas y ensayos europeos, establecí hace años la distribución geográfica de las denominaciones del arco iris en nuestro entorno y puse de relieve su utilidad para trazar la historia de las palabras y las creencias asociadas a este fenómeno meteorológico (García Mouton 1984). En aquel momento los datos del Atlas Lingüístico de la Península Ibérica (ALPI) no estaban accesibles. No cabe duda de que, si el ALPI se hubiera editado con normalidad, habría cumplido el papel reservado a los atlas de gran dominio, proporcionar un marco general a los atlas posteriores, pero las circunstancias invirtieron el orden natural de la investigación y, a finales del siglo pasado, se contaba con varios atlas de pequeño dominio, pero se carecía del atlas general. La situación ha cambiado, y ahora disponemos de esos materiales del $A L P I$, de los de atlas publicados desde entonces ${ }^{3} \mathrm{y}$ de trabajos monográficos pertenecientes a distintas áreas (Alinei 1981, 1983, 1984; Alinei \& Benozzo 2011; Martines 2012).

Estas páginas pretenden valorar la aportación del $A L P I$ para conocer el léxico relativo al arco iris, completar una visión de conjunto y reivindicar el valor etnográfico de sus materiales. Los mapas que componen el primer y único volumen del atlas, publicado en 1962, indujeron a que fuese considerado un atlas casi exclusivamente fonético. Sin embargo, ya Tomás Navarro Tomás reivindicó su condición de atlas etnográfico, aunque no lo explicitase en el título. ${ }^{4}$ De hecho, el equipo de la Sección de

\footnotetext{
${ }^{2}$ Trabajé entonces con los atlas regionales dirigidos por Manuel Alvar: Atlas Lingüístico y Etnográfico de Andalucía (ALEA, IV, 860); Atlas Lingüístico y Etnográfico de Aragón, Navarra y Rioja (ALEANR X, 1336); Atlas Lingüístico y Etnográfico de las Islas Canarias (ALEICan II, 762), y los materiales inéditos para el proyectado Atlas Lingüístico de España y Portugal (ALEP), el que sería el Atlas Lingüístico y Etnográfico de Cantabria (ALECant) y el Léxico de los Marineros Peninsulares (LMP 123). Para el catalán, con los atlas de Antoni Griera: Atlas Lingüístic d'Andorra (ALA, 119); Atlas Lingüístic de Catalunya (ALC I, 144) y Atlas Lingüistic de la Vall d'Aran (ALVA, 142.)

${ }^{3}$ El Atlas Lingüístico del Bierzo (ALBi 85) de Manuel Gutiérrez Tuñón, y para la zona castellana, la menos investigada, el Atlas Lingüístico de Castilla y León ( $A L C y L)$ de Manuel Alvar, el Atlas Lingüístico y etnográfico de Castilla-La Mancha (ALeCMan 971) de Pilar García Mouton y Francisco Moreno Fernández y el Atlas Dialectal de Madrid (ADiM 950) de Pilar García Mouton e Isabel Molina Martos; para el gallego, el Atlas Lingüístico Galego (ALGa IV, 91) de Constantino García y Antón Santamarina, y, para el catalán, el Atles Lingüístic del Domini Català (ALDC 678) de Joan Veny y Lídia Pons i Griera.

${ }^{4}$ Dejó escrito: "Para la sección de léxico resultó de gran ayuda el Atlas italo-suizo de Jaberg y Jud, cuyos volúmenes empezaron a aparecer por esa fecha. Adoptamos su organización por temas etnográficos
} 
Filología del madrileño Centro de Estudios Históricos había tenido ocasión de aprovechar las enseñanzas de los directores del atlas italosuizo, el AIS, como prueba el cuestionario, organizado por campos léxicos, entre los que figura el dedicado a los fenómenos atmosféricos. ${ }^{5}$ En la página 5 del Cuaderno II, entre Chaparrón y Niebla, figura la pregunta 433 Arco iris. Sus respuestas reúnen los nombres del fenómeno atmosférico en las 527 localidades encuestadas, y también las informaciones adicionales y los refranes relacionados con él que los encuestadores registraron.

\section{Los nombres del arco iris}

La tradición considera el arco iris como un fenómeno excepcional en el que intervienen entes sobrenaturales, muy ligado a la actividad agrícola, porque anuncia lluvias o el final del mal tiempo. En el marco de su teoría de la continuidad, Mario Alinei (Alinei \& Benozzo 2011: 3-5) clasificó los icónimos ${ }^{6}$ del arco iris en cuatro grupos, desde los más recientes, que reflejan 1) una interpretación mágico-religiosa cristiana o musulmana del arco iris y 2) una interpretación mágico-religiosa asociada a divinidades o a seres míticos antropomórficos precristianos o preislámicos, a los más alejados en el tiempo, que reflejan 3) la interpretación del arco como un animal gigantesco, o parte de él, y 4) una subclase constituida por el icónimo de un animal que bebe agua y la devuelve en forma de lluvia.

Las denominaciones que recoge el $A L P I$ caben en esta clasificación, especialmente en los dos primeros grupos. La mayor parte corresponde a nombres genéricos basados en la forma curva del arco iris, cuyos principio y fin tocan la tierra: arco sobre todo, pero también puente, ratlla, cinto, cinta, a los que se une el nombre

\footnotetext{
siguiendo el orden de fenómenos atmosféricos, accidentes geográficos, flora, fauna, cuerpo humano, familia, hogar, labores agrícolas, oficios artesanos, herramientas, animales domésticos, etc. Sobre esta base, el ALPI hubiera podido llamarse Atlas lingüístico y etnográfico, como de hecho lo es, aunque no pareciera indispensable indicarlo en el título" (Navarro Tomás 1975: 12-13).

${ }^{5}$ En 1949, en carta a Sever Pop (s.a.: 429), Navarro Tomás decía: "Tratamos de recoger y aprovechar las experiencias del Atlas de la France y del de (sic) Italien und Südschweiz. Este último sirvió como principal modelo para agrupación de materias en el cuestionario de lexicografía".

${ }^{6}$ Llama icónimo a una palabra preexistente que, en el momento de la lexicalización y de un nuevo referente, presta su significado a la nueva palabra.
} 
del ente con el que se relaciona. Lo normal es la referencia a entes femeninos precristianos: arco de la vieja, arco da vella, arco da velha y arco iris. Esta última denominación perdió pronto, si es que alguna vez la tuvo para los hablantes, su vinculación con Iris, la mensajera de los dioses, cuyo nombre lexicalizado nadie asocia a una túnica o un cinturón de colores. ${ }^{7}$ Aunque actualmente para muchos el arco iris simboliza el pacto bíblico de Dios con Noé tras el diluvio, sus raíces paganas no siempre traslucen una interpretación propicia. En algunos sitios lo retan, hacen ademán de cortarlo y, si alguien se atreve a burlarse de él, debe temer sus castigos. ${ }^{8}$

En el norte y el oeste peninsular se localizan los nombres precristianos: el asturiano $\operatorname{arco} d a(s)$ vella(s), arco das viechas es arco da(s) vella(s) en el occidente de El Bierzo y en Galicia y arco da velha en territorio portugués, desde donde pasó a las islas Canarias como arco (de) la vieja, uno de sus portuguesismos encubiertos (García Mouton 1984: 171). La vieja, la vetula que tanto estudió Gerhard Rohlfs (1979: 79103), corresponde a la vieja telúrica cuya imagen se mantiene, entre otros lugares, en los montes de León como la güela o la vieya ${ }^{9}$ y pervive en alguno de los nombres gallegos de la luciérnaga, como a vella facendo papas (Hernández \& Molina 1999). En cierto modo, también podría considerarse precristiano arco iris que, con arco, es general en amplias zonas castellanas, como probables sustitutos de los arco del cielo y arco celeste habituales en nuestros clásicos (García Mouton 1984: 177), semejantes al francés arc-en-ciel, aunque arco iris es forma culta, tardía en castellano, difundida por la instrucción y cuyo actual arraigo se debe en gran medida a la pérdida de las creencias populares tradicionales asociadas a este fenómeno atmosférico.

En etapas posteriores, las antiguas denominaciones se cristianizaron $y$, sin embargo, la vieja casi nunca fue sustituida por la Virgen ${ }^{10}$ - hay un caso palentino de arco la Virgen - o por alguna santa, sino por referencias a Dios - arco del Señor, arco

\footnotetext{
${ }^{7}$ Aparecen casos aislados en Zamora de cinto de Dios (Fariza 347), la cinta viella (San Martín de Castañeda 338) y, en Bragança, Portugal, cinta de la raposa (Duas Igrejas 225).

${ }^{8}$ Por eso, en 121 Palas de Rei (Lugo), le dicen: "Marco da vella vaite de ahí que as nenas bonitas non son para ti, que son pro rei que vén ahí" y, en 134 Bueu (Pontevedra), "Arco da vella vaite de ahí, que andan os porcos a fozar en ti". En 146 O Bolo (Ourense), lo interpelan así: "Arco de vellas, revolve tres pedras» y el encuestador añadió que es lo que "Le dicen para que cese de llover".

${ }^{9}$ Los niños creen que vive en una cueva en el monte y que les regala pan, queso y otros restos de comida - que llaman pan de paxarines o pan de la raposa- a los campesinos que, al volver a casa, se los llevan de su parte a ellos (Bartolomé Pérez \& Gancedo 2014).

${ }^{10}$ En San Miguel de las Dueñas, León, lo llaman arco iris y corona de la Virgen (ALBi 85).
} 
/arquito de Dios, arcu /arco de Cristo-y, sobre todo, según las zonas, a determinados santos - arco de Santiago, arco de san Martín, arco de san Juan, arco de san Pedro-. Excepcionalmente aparece alguna referencia bíblica, un caso de arcu de Noé en Cantabria, otro de arco da aliansa en Portugal, pero en términos generales el cristianismo medieval privilegió el culto a santos varones y masculinizó la presencia femenina que había detrás del arco. ${ }^{11}$

\section{Distribución}

Los nombres del arco iris que recoge el ALPI pertenecen a las distintas variedades romances de la península y Baleares y, como era de esperar, vienen a coincidir con los de los atlas regionales (García Mouton 1984: 176). Su distribución por áreas es la siguiente:

Arco da(s) vella(s), de vella en el occidente asturiano y berciano, Galicia y Portugal, con casos aislados en la franja española que linda con Portugal, algún caso de marco de vello (Pontevedra, A Golada 128), marco da vella (Lugo, Palas de Rei 121), marco das vellas (Orense, Rairiz de Veiga 148) y barco da vella (Pontevedra, Nigrán 136).

Arco iris y arco, generales en las zonas castellanas, alcanzan la Andalucía oriental. Arco del Señor, denominación central castellana

Arco de Santiago, denominación extremeña

Arco de san Juan, denominación navarra y aragonesa, con un caso de barra san Juan (605)

Arco de san Pedro, denominación aragonesa, aparece muchas veces en combinación con la anterior.

Arc, arquet, arquico, rall, ralla, ratlla de sant Martí, arco de san Martín, denominaciones propias del aragonés oriental y de las zonas catalanohablantes y de su influencia

\footnotetext{
${ }^{11}$ En Portugal, junto a arco da velha, hay ejemplos de arco (da) Virgem y arco da Nossa Senhora.
} 


\section{Aportaciones del ALPI}

Las aportaciones del $A L P I$ se basan en determinados aspectos derivados de la observación del arco iris:

4.1. En ocasiones no aparece un solo arco en el cielo, sino dos. Entonces los hablantes consideran que el más luminoso es el principal y el más desvaído, el secundario, y les dan nombres diferentes. En 326, Páramo del Sil (León), Ilaman arcu de las viyechas al más grande y lo oponen al arcu las novas, como en 126 Sober (Lugo), donde el arco das vellas es diferente al arco das novas. ${ }^{12}$

Según Joan Amades (1939: 115-115) y Antoni Griera (1947: 263), los dos arcos representaban una lucha antigua entre el bien y el mal, por eso en catalán llaman arc del dimoni al arco secundario. Cuenta la leyenda que san Martín y el demonio compitieron para ver quién hacía un arco mejor y más brillante. San Martín logró hacer un arco de cristal, grande y de colores vivos, mientras que el demonio, que tardó más, hizo uno pequeño, de colores apagados, que se desdibujaba fácilmente porque era de hielo ${ }^{13}$. Los datos catalanes del ALPI no recogen esta creencia, pero el Atles Linguístic del Domini Català (III, mapa 678) registra del dimoni para el segundo arco en un solo punto (34, Santa Maria de Corcó) y el DCVB recoge, sin localización, arc del dimoni o arc suís para el "arc secundari concèntric a l'arc de St. Martí i de coloració més dèbil que aquest". Sin embargo, los cuestionarios aragoneses del ALPI documentan casi sistemáticamente $\operatorname{arco}(d e)$ san Juan para el arco grande y arco (de) san Pedro para el más pequeño. ${ }^{14}$

\footnotetext{
${ }^{12}$ En 126 Sober (Lugo), Aníbal Otero anotó: "Hay un arco que dice azul (o das vellas) y outro que ten unhas cintas amarillas», arco das novas. En 131 Vilanova de Arousa (Pontevedra), recogió que el arco da vella "es para pasar el aire por debajo" y que llaman bicho al que "tiene colores como el arco iris, pero no sube apenas del horizonte; echa el viento de alí para acó".

${ }^{13}$ En Luxemburgo, el arco mejor se atribuye a Dios o a la Virgen y el menos visible, al diablo, que imita mal lo que hace Dios, por eso lo llaman arc-en-ciel du diable (Gaidoz 1890-91: 166). Una reminiscencia de la primitiva oposición se recoge aún a pocos kilómetros de Madrid: "Cuando llueve y hace frío, sale el arco del judío; cuando llueve y hace sol, sale el arco del Señor" (ADiM 950, M9); también en la provincia de Ciudad Real, en CR 605 llaman arco del Señor al grande y arco del judío, al pequeño (ALeCMan 971).

${ }^{14}$ Así en Huesca, 611 (Alquézar), 614 (Sant Esteve de Llitera), 615 (Fonz), 616 (Peralta de Alcofea); en Zaragoza, 621 (Monegrillo), 623 (Cadrete), 625 (Codos) y, en Teruel, 631 (Segura de los Baños), 632 (Alloza) y 636 (Alfambra).
} 
4.2. A veces el arco puede aparecer roto, de manera que solo deja ver el arranque y el final, y entonces lo llaman pata de cabra. En Ávila, en 451 (La Horcajada), medio arco es pata de cabra, lo mismo que en Jaén, 511 (Menjíbar); en Albacete, en 485 (Higueruela), si está partido en dos, es pata (d)e cabra; en Zaragoza, en 620 (Boquiñeni), verderol si solo se ve la parte que toca tierra; en Valencia, en 769 (Rafelbunyol), es cabreta cuando está incompleto; y en las Baleares, en 796 (Sineu), arc de sant Martí, el completo, y coll de sa cabra, si está roto. También en Castilla-La Mancha el ALeCMan recoge casos de patacabra: en CR 408 llaman arco del Señor al que sale al este y patacabra al que sale al oeste; en CR 606 lo llaman arco iris y patacabra; mientras que en CR 608 y 610 especifican que patacabra es medio arco.

Según Rohlfs (1979: 102-103), la cabra, y también el zorro, ${ }^{15}$ son animales tras los que suele esconderse la vieja y aparecen en los nombres de otros fenómenos atmosféricos como la 'vibración del aire' o la 'niebla', que en provenzal llaman crabo. La cabra vendría a ser la forma animal de la bruja o del diablo, de manera que estos pata de cabra, cabreta y coll de sa cabra estarían vinculados con los arco da vella del norte y el este peninsular.

4.3. Finalmente, dependiendo de su orientación y de si aparece por la mañana o por la tarde, la tradición campesina interpreta que el arco anuncia lluvia buena o mala para el campo. ${ }^{16}$ En muchos casos el informante apoyó ese conocimiento predictivo en un refrán que transmite la experiencia de su comunidad.

4.3.1. En una franja occidental de norte a sur, el arco iris trae agua y remedia la sed.

327 Omañón (León). Arco meiruelu, ni mal tiempu ni buenu

330 Pontedo (León). Cuando el arco de san Juan baja beber agua el río, señas de llover o nevar o de hacer frío.

\footnotetext{
${ }^{15} \mathrm{~V}$. las referencias la raposa 'zorra', en las notas 7 y 8.

${ }^{16}$ En Dragonte (ALBi 85) dicen "arco de vella, tempo da merda".
} 
352 Hinojosa (Salamanca). Arco azul y verde, no morirás de sede.

353 Retortillo (Salamanca). Arco verde, no te morirás de sede.

374 Valle de Santa Ana (Badajoz). Cuando el arco vieres, no morirás de sede.

376 Valencia de Mombuey (Badajoz). Cuando el arco la vieja viene en tarde, no viene en balde.

377 Calera de León (Badajoz). El que arco ve, naide se muere de sed.

531 Lebrija (Sevilla). El que arco iris ve, no se seca de sed.

705 Llo (Cerdaña, Pyrénées Orientales). Arquet de Sant Martí, bouer, torna-te'n a dormir.

733 Sant Bartomeu del Grau (Barcelona). Arc de Sant Martí al matí, pareu-li el bací; si surt al vespre, pareu-li la testa.

793 Calvià (Baleares). S'arc de sent Martí, si surt es de matí, fé es teu camí; i si surt de capvespre, treu es cap a sa finestra.

4.3.2. Cuando el arco sale al oeste o poniente, puede anunciar lluvia, y entonces el refrán dictamina que es momento de recoger los bueyes, la yunta, el par, las vacas o el arado y volver a casa. En algunos sitios, se añade otro refrán que indica qué hacer cuando el arco se ve al este.

348 Pego (Zamora). Arco al poniente, echa el arado y vente.

351 Campo de Peñaranda (Salamanca). Arco hacia Ledesma, echa el arado y vete a la taberna. [Ledesma queda al oeste de Peñaranda].

355 Serradilla del Arroyo (Salamanca). Agua al poniente, agua al amaneciente 371 Almendral (Badajoz), 522 Almonte (Huelva), 529 El Coronil (Sevilla), 530 Saucejo (Sevilla), 536 Cuevas del Becerro (Málaga). Arco al poniente, suelta los bueyes y vente.

468 Los Yébenes (Toledo). Arco al puniente, suelta los bueyes y vente. / Arco en el río, jornal perdido [El río queda al este del pueblo].

475 Puebla de Don Rodrigo (Ciudad Real). Si en mayo ves el arco salir al puniente, suelta los bueyes y vente. 
476 Alcolea de Calatrava (Ciudad Real). Arcos (sic) al puniente, suelta los bueyes y vente.

479 Fuencaliente (Ciudad Real). Arco al puniente, suelta los bueyes y vente. 480 El Viso del Marqués (Ciudad Real), 506 La Carlota (Córdoba), 514 Pegajalar (Jaén). Arco al puniente, suelta la yunta y vente.

481 Carrizosa (Ciudad Real). Arco al puniente, déjate la yunta y vente.

504 Adamuz (Córdoba), 537 Peñarrubia (Málaga). Arco al poniente, suelta la yunta y vente.

507 Doña Mencía (Córdoba). Arco al puniente, suelta la yunta y vente. / Arco al levante, estate mañana sol picante.

510 Puerta del Segura (Jaén). Arco al poniente, suelta el par y veste.

526 Bormujos (Sevilla). Arco al poniente, suelta los bueyes y vente. / Arco Lebrija, agua fija. [Lebrija queda al sur de Bormujos].

528 Gilena (Sevilla). Arco al poniente, suelta las vacas y vente.

554 Suflí (Almería). Arco al poniente, carga el arado y vente.

4.3.3. En otros lugares, el arco que se ve al este, levante o saliente, anuncia lluvia y entonces hay que recoger los bueyes, la yunta o el arado y volver a casa. Hay puntos en los que se añade un segundo refrán que indica qué hay que hacer en caso de que el arco salga al oeste. En dos localidades andaluzas (503 y 554), un arco al este se interpreta como señal de buen tiempo.

372 Fuente del Maestre (Badajoz), 373 Hornachos (Badajoz), 375 Ahillones (Badajoz). Arco al saliente, deja los bueyes y vente.

464 Cebolla (Toledo). Arco al saliente, coge los bueyes. / Arco al saliente, coge los bueyes y vente.

477 Villarrubia de los Ojos (Ciudad Real). Arco al saliente, desunce las yuntas y vente.

501 Pedroche (Córdoba). Arco al saliente, suelta la yunta y vente.

503 Villaviciosa (Córdoba). Arco al levante, tiempo abonante / Arco al saliente, suelta la yunta y vente. 
505 Hornachuelos (Córdoba). Arco iris al saliente, coge la yunta y vente.

507 Doña Mencía (Córdoba) [Arco al puniente, suelta la yunta y vente.]/ Arco al levante, estate mañana sol picante.

527 Fuentes de Andalucía (Sevilla). Arco al saliente, suéltalos y vente.

532 El Bosque (Cádiz). Arco al naciente, suelta los bueyes y vente.

554 Suflí (Almería). [Arco al poniente, carga el arado y vente.] / Arco al levante, descarga el arado y estate.

En general, los atlas españoles documentan pocos refranes (García Mouton 2012), ${ }^{17}$ por lo que se puede afirmar que el $A L P I$ resulta especialmente rico en este sentido. Es cierto que los cuestionarios de los atlas lingüísticos no suelen prever preguntas específicas dedicadas a refranes, porque no constituyen ítems lingüísticos comparables entre los distintos puntos de una red, pero los investigadores conocen su interés y saben en qué momento de la encuesta pueden surgir, sobre todo al tratar cuestiones relativas a fenómenos meteorológicos como el arco iris, el cielo emborregado o los arreboles del amanecer y del atardecer (Gargallo Gil 2006, 2011; García Mouton 2012; ParemioRom). Esto explica que la recogida de refranes sea desigual en los atlas y dependa no solamente de la información que recuerden los informantes, sino también del interés de quienes hacen la encuesta y elaboran los materiales. $^{18}$

En el ALPI resulta evidente que hay zonas -Navarra, Aragón, Cataluña (tres puntos), Valencia, Galicia (tres puntos) - en las que casi no se recogieron refranes para el arco iris, sí para otros conceptos, de manera que es probable que allí sean menos frecuentes. Sin embargo, como se puede apreciar en el apéndice que sigue, llama la atención la sensibilidad de Aurelio M. Espinosa hijo ${ }^{19}$ al apuntar refranes relativos al arco iris en sus cuestionarios.

\footnotetext{
${ }^{17}$ Los atlas regionales de Manuel Alvar, a pesar de su carácter etnográfico, no ofrecen muchos refranes. El ALEA aporta unos pocos; el ALEICan y el ALEANR, algunos más. En el ALeCMan y en el ADiM se puso especial interés en recoger este tipo de etnotexto fosilizado.

${ }^{18}$ Los primeros atlas no prestaron gran atención a estos contenidos, pero, a partir del movimiento Wörter und Sachen, trataron de incorporar todo lo que la cultura popular podía aportar como complemento de la información lingüística.

${ }^{19}$ Espinosa incluye en bastantes de sus encuestas una lista final de refranes.
} 
Dialectologia. Special issue, IX (2021), 135-149.

ISSN: 2013-2247

\section{Bibliografía}

Atlas Lingüísticos

ADiM = GARCíA Mouton, Pilar \& Isabel Molina MARTOS (2015 -) Atlas Dialectal de Madrid (ADiM) <http://adim.cchs.csic.es/>

ALA = GRIERA, Antoni (1969) Atlas Lingüístic d'Andorra, Andorra .

ALBI = GUTIÉRREZ TUÑóN, Manuel (dir.) \& Alicia FONTEBOA LóPEZ (coord.) (1996) Atlas Lingüístico del Bierzo, vol. I, Léxico (I); GutiéRrez TuÑón, Manuel (dir.) \& Ma José RodríGUez Malmierca (coord.) (2002) Atlas Lingüístico del Bierzo, vol. II, Léxico (II), Ponferrada: Instituto de Estudios Bercianos.

ALC = GRIERA, Antoni (1923) Atlas Lingüístic de Catalunya, Barcelona: Institut d'Estudis Catalans; (1962) Barcelona: Polígrafa.

ALCyL = ALVAR, Manuel (1993) Atlas Lingüístico de Castilla y León, Salamanca: Junta de Castilla y León, Consejería de Educación y Cultura.

$A L D C=$ VeNY, Joan \& Lídia PONS i GRIERA (2001-2018) Atles Lingüístic del Domini Català (ALDC), Barcelona: Institut d' Estudis Catalans. <https://aldc.espais.iec.cat>

ALEA = ALVAR, Manuel, con la col. de Antonio LLORENTE \& Gregorio SALVADOR (1961-1973) Atlas Lingüístico y Etnográfico de Andalucía, Granada: Universidad de Granada - CSIC.

ALEANR = ALVAR, Manuel, con la colaboración de Tomás BUESA, Antonio LLORENTE \& Elena ALVAR (1979-1993) Atlas Lingüístico y Etnográfico de Aragón, Navarra y Rioja. Madrid: Institución Fernando el Católico - CSIC.

ALEICan = ALVAR, Manuel (1975-1978) Atlas Lingüístico y Etnográfico de las Islas Canarias, Las Palmas: Ediciones del Excmํ Cabildo Insular de Gran Canaria.

ALECant = Alvar, Manuel, con la col. de Carlos Alvar \& José Antonio MaYoral (1995) Atlas Lingüistico y Etnográfico de Cantabria, Madrid: Arco Libros.

ALeCMan = GARCía MoUton, Pilar \& Francisco MoReno Fernández (2003-) Atlas Lingüístico y etnográfico de Castilla-La Mancha (2003-) <https://www.linguas.net/alecman/>

aLGa = GarcíA, Constantino \& Antón SANTAMARINA (dirs.) (1990-2020) Atlas Lingüístico Galego, Santiago de Compostela: Fundación Barrié / Servizo de Publicacións da Universidade de Santiago de Compostela. 
ALPI $=$ [NAvarro Tomás, Tomás (dir.)] (1962) Atlas Lingüístico de la Península Ibérica, I. Fonética, Madrid: CSIC. <http://ALPI.csic.es/es/publicaciones>

ALPI edición digital = GARCíA Mouton, Pilar (coord.), Inés FeRnÁNDEZ-ORDóÑEZ, David HeAP, Maria Pilar Perea, João Saramago \& Xulio Sousa (2016-) ALPI-CSIC, edición digital del Atlas Lingüístico de la Península Ibérica, NAVARRo TOMÁs, Tomás (dir.), Madrid: CSIC $<$ www.ALPI.csic.es>

ALVA = GrIERA, Antoni (1973) Atlas Lingüístic de la Vall d'Aran, Barcelona: Ed. Polígrafa.

LMP = ALVAR, Manuel (coord.) (1985-1989) Léxico de los marineros peninsulares, Madrid: Arco Libros.

PAREMIOROM = Paremiología romance: refranes meteorológicos y territorio, GARGALLO GIL, José Enrique (IP) <http://stel.ub.edu/paremio-rom/es/search/node/arco\%20iris>

Estudios

AlINEl, Mario (1981) "Tre studi onomasiologici sull'arcobaleno. Presentazione", Quaderni di Semantica, 2, 51-53.

AlINEl, Mario, "L'Arc-en-ciel" (1983), in Atlas Linguarum Europae. Commentaires [mapas I.6I.9], vol. I, premier fasc., Van Gorcum: Assen, 47-80.

ALINEI, Mario (1984) "I nomi dell'arco baleno in Europa. Una ricerca nel quadro dell'ALE", in Diacronia, sincronica e cultura. Saggi linguistici in onore di Luigi Heilmann, Brescia: La Scuola, 365-384.

Alinel, Mario \& Francesco Benozzo (2011) "A pré-história dos nomes do arco-íris", in Arqueologia Etimológica. Três Estudos acerca da Continuidade Lingüístico-Cultural do Paleolítico, Lisboa: Apenas Livros, 3-14.

AMADES, Joan (1930) "Astronomia i meteorologia populars", Butlletí de Dialectologia Catalana, XVIII, abril-junio, 114 \& ss.

BARTOlomé Pérez, Nicolás \& Emilio GANCEDo (12 de enero de 2014) “La bruja buena de los montes leoneses", Diario de León, 12.

$D C V B=$ ALCOVER, Antoni Maria \& Francesc de Borja Moll (1962) Diccionari català-valenciàbalear, Ed. Moll. <https://DCVB.iec.cat/>

GarCía MoUton, Pilar (1984) "El arco iris: Geografía lingüística y creencias populares", Revista de Dialectología y Tradiciones Populares, 39, 169-190. <http://adim.cchs.csic.es/es/publicaciones>

García Mouton, Pilar (2012) “Refranes meteorológicos en el Atlas Lingüístico (y etnográfico) de 
Dialectologia. Special issue, IX (2021), 135-149.

ISSN: 2013-2247

Castilla-La Mancha", Géolinguistique, 127-138. <http://digital.csic.es/handle/10261/123075>

GRIERA, Antoni (1947) Tresor de la Llengua, de les Tradicions i de la Cultura popular de Catalunya, Barcelona: Ed. Polígrafa.

Gargallo GIL, José Enrique (2006) “Quan surt la ratlla de Sant Martí... Refranes romances del arco iris, meteorología y cultura popular", Quaderni di Semantica, XXVII, 1-2/2006 (Studi in onore di Mario Alinei), 301-319.

GARGALlo GIL, José Enrique et al. (coord.) (2011) I proverbi meteorologici ai confini dell'Europa romanza, Alessandria: Edizioni dell’ Orso, 2011.

HERNÁNDEZ, Esther \& Isabel MolinA (1999) “Los nombres de la luciérnaga en la geografía lingüística de España y América”, Géolinguistique, 8, 83-117. <https://www.researchgate.net/publication/282112591>

MARTINES, Josep (2012) “La integració conceptual o blending i l'arc de Sant Martí. Vers una semàntica cognitiva diacrònica i cultural", eHumanista/IVITRA 2, 240-270.

<https://www.ehumanista.ucsb.edu/sites/secure.Isit.ucsb.edu.span.d7_eh/files/sitefiles/ivitra /volume2/11Martines20240-270.pdf>

POP, Sever (s.a.) La Dialectologie, I, Gembloux: Imprimerie J. Duculot.

ROHLFS, Gerhard (1939) "Romanischer Volksglaube um die Vetula", Archiv für das Studium der neueren Sprachen 175, 65-75. [Traducido al español y anotado por Manuel ALVAR como "El problema de la "Vetula"” en G. RoHLfS (1970), Estudios sobre el léxico románico, Madrid: Gredos, 70-103]. 


\section{APÉNDICE}

Refranes sobre el arco iris en el ALPI

- Recogidos por Aníbal Otero

121 Palas de Rei (Lugo). Marco da vella vaite de ahí que as nenas bonitas non son para ti, que son pro rei que vén ahí.

134 Bueu (Pontevedra). Arco da vella vaite de ahí, que andan os porcos a fozar en ti.

146 O Bolo (Ourense). Arco de vellas, revolve tres pedras.

-Recogidos por Aurelio M. Espinosa hijo

327 Omañón (León). Arco meiruelu, ni mal tiempu ni buenu

330 Pontedo (León). Cuando el arco de san Juan baja beber agua el río, señas de llover o nevar o de hacer frío.

348 Pego (Zamora). Arco al poniente, echa el arado y vente.

351 Campo de Peñaranda (Salamanca). Arco hacia Ledesma, echa el arado y vete a la taberna.

352 Hinojosa (Salamanca). Arco azul y verde, no morirás de sede.

353 Retortillo (Salamanca). Arco verde, no te morirás de sede.

355 Serradilla del Arroyo (Salamanca). Agua al poniente, agua al amaneciente

371 Almendral (Badajoz). Arco al poniente, suelta los bueyes y vente.

372 Fuente del Maestre (Badajoz). Arco al saliente, deja los bueyes y vente.

373 Hornachos (Badajoz). Arco al saliente, deja los bueyes y vente.

374 Valle de Santa Ana (Badajoz). Cuando el arco vieres, no morirás de sede.

375 Ahillones (Badajoz). Arco al saliente, deja los bueyes y vente.

376 Valencia de Mombuey (Badajoz). Cuando el arco la vieja viene en tarde, no viene en balde.

377 Calera de León (Badajoz). El que arco ve, naide se muere de sed.

464 Cebolla (Toledo). Arco al saliente, coge los bueyes. / Arco al saliente, coge los bueyes y vente. 468 Los Yébenes (Toledo). Arco al puniente, suelta los bueyes y vente. / Arco en el río, jornal perdido

475 Puebla de Don Rodrigo (Ciudad Real). Si en mayo ves el arco salir al puniente, suelta los bueyes y vente.

476 Alcolea de Calatrava (Ciudad Real). Arcos (sic) al puniente, suelta los bueyes y vente.

477 Villarrubia de los Ojos (Ciudad Real). Cuando llueve y hace sol, sale el arco del Señor. / Arco al saliente, desunce las yuntas y vente.

479 Fuencaliente (Ciudad Real). Cuando llueve y hace sol, sale el arco del Señor. /Arco al puniente, suelta los bueyes y vente.

480 El Viso del Marqués (Ciudad Real). Arco al puniente, suelta la yunta y vente. 
Dialectologia. Special issue, IX (2021), 135-149.

ISSN: 2013-2247

481 Carrizosa (Ciudad Real). Arco al puniente, déjate la yunta y vente.

501 Pedroche (Córdoba). Arco al saliente, suelta la yunta y vente.

503 Villaviciosa (Córdoba). Arco al levante, tiempo abonante / Arco al saliente, suelta la yunta y vente.

504 Adamuz (Córdoba). Arco al poniente, suelta la yunta y vente.

505 Hornachuelos (Córdoba). Arco iris al saliente, coge la yunta y vente.

506 La Carlota (Córdoba). Arco al puniente, suelta la yunta y vente.

507 Doña Mencía (Córdoba). Arco al puniente, suelta la yunta y vente. / Arco al levante, estate mañana sol picante.

510 Puerta del Segura (Jaén). Arco al poniente, suelta el par y veste.

514 Pegajalar (Jaén). Arco al puniente, suelta la yunta y vente.

522 Almonte (Huelva). Arco al poniente, suelta los bueyes y vente.

526 Bormujos (Sevilla). Arco al poniente, suelta los bueyes y vente. / Arco Lebrija, agua fija

527 Fuentes de Andalucía (Sevilla). Arco al saliente, suéltalos y vente.

528 Gilena (Sevilla). Arco al poniente, suelta las vacas y vente.

$529 \mathrm{El}$ Coronil (Sevilla). Arco al poniente, suelta los bueyes y vente.

530 Saucejo (Sevilla). Arco al poniente, suelta los bueyes y vente.

531 Lebrija (Sevilla). El que arco iris ve, no se seca de sed.

532 El Bosque (Cádiz). Arco al naciente, suelta los bueyes y vente.

536 Cuevas del Becerro (Málaga). Arco al poniente, suelta los bueyes y vente.

537 Peñarrubia (Málaga). Arco al poniente, suelta la yunta y vente.

554 Suflí (Almería). Arco al poniente, carga el arado y vente. / Arco al levante, descarga el arado y estate.

- Recogidos por Manuel Sanchis Guarner

705 Llo (Cerdanya, Pyrénées Orientales). Arquet de Sant Martí, bouer, torna-te'n a dormir.

733 Sant Bartomeu del Grau (Barcelona). Arc de Sant Martí al matí, pareu-li el bací; si surt al vespre, pareu-li la testa.

793 Calvià (Baleares). S'arc de sent Martí, si surt es de matí, fé es teu camí; i si surt de capvespre, treu es cap a sa finestra. 